Abelardo Gil-Fournier (FAMU, Prague) /Jussi Parikka (FAMU, Prague \& University of Southampton)

article manuscript (not proofread) - August 2019

\title{
"Visual Hallucination of Probable Events", or, On Environments of Images and Machine Learning
}

\section{Introduction}

Contemporary images come in many forms but also, importantly, in many times. Screens, interfaces, monitors, sensors and many other devices that are part of the infrastructure of knowledge build up many forms of data visualisation in so-called real-time. While data visualisation might not be that new of a technical form of organisation of information as images, it takes a particularly intensive temporal turn with networked data that has been discussed for example in contexts of financial speculation. ${ }^{1}$ At the same time, these imaging devices are part of an infrastructure that does not merely observe the microtemporal moment of the "real", but unfolds in the now-moment. In terms of geographical, geological and broadly speaking environmental monitoring, the now moment expands in to near-future scenarios in where other aspects, including imaginary are at play. Imaging becomes a form of nowcasting, exposing the importance of understanding change changing.

Here one thinks of Paul Virilio and how "environment control" functions through the photographic technical image. In Virilio's narrative the connection of light (exposure), time and space are bundled up as part of the general argument about the disappearance of the spatio-temporal coordinates of the external world. From the realspace we move to the 'real-time' interface ${ }^{2}$ and to analysis of how visual management detaches from the light of the sun, the time of the seasons, the longue duree of the planetary qualitative time to the internal mechanisms of calculation that pertain to electric and electronic light. Hence, the photographic image that is captured prescribes for Virilio the exposure of the world: it is an intake of time, and, an intake of light. Operating on the world as active optics, these intakes then become the defining temporal frame for how environments are framed and managed through "operational images", to use Harun Farocki's term ${ }^{3}$, and which then operationalize how we see geographic spaces too. The time of photographic development (Niepce), or "cinematographic resolution of movement" (Lumière), or for that matter the "videographic high definition of a 'real-time' representation of appearances"4 are part of Virilio's broad chronology of time in technical media culture.

But what is at best implied in this cartography of active optics is the attention to mobilization of time as predictions and forecasts. For operations of time and production of times move from meteorological forecasting to computer models, and

1 Sean Cubitt, Three Geomedia, in: Ctrl-Z 7, 2017; http://www.ctrlz.net.au/articles/issue-7/cubitt-three-geomedia/ [Zugriff 29.08.2018].

${ }^{2}$ Paul Virilio, Polar Inertia, London-Thousand Oaks-New Delhi, 2000, S. 55.

${ }^{3}$ Harun Farocki, Phantom Images, in: Public 29, 2004, S. 12-24, hier S. 17.

${ }^{4}$ Ebenda, S.61. 
from computer models to a plethora of machine learning techniques that have become another site of transformation of what we used to call photography. Joanna Zylinska names this generative life of photography as its nonhuman realm of operations that rearranges the image further from any historical legacy of anthropocentrism to include a variety of other forms of action, representation and temporality. ${ }^{5}$ The techniques of time and images push further what counts as operatively real, and what forms of technically induced hallucination - or, in short, in the context of this paper, machine learning - are part of current forms of production of information.

Also in information society, digital culture, images persist. They persist as markers of time in several senses that refer not only to what the image records - the photographic indexicality of a time passed nor the documentary status of images as used in various administrative and other contexts - but also what it predicts. Techniques of machine learning are one central aspect of the reformulation of images and their uses in contemporary culture: from video prediction of the complexity of multiple moving objects we call traffic (cars, pedestrians, etc.) to satellite imagery monitoring agricultural crop development and forest change. Such techniques have become one central example of where earth's geological and geographical changes become understood through algorithmic time, and also where for instance the very rapidly changing vehicle traffic is treated alike as the much slower earth surface durations of crops. In all cases, a key aspect is the ability to perceive potential futures and fold them into the real-time decision-making mechanisms.

The computational microtemporality takes a futuristic turn; algorithmic processes of mobilizing datasets in machine learning become activated in different institutional context as scenarios, predictions and projections. Images run ahead of their own time as future-producing techniques.

Our article is interested in a distinct technique of imaging that speaks to the technical forms of time-critical images: Next Frame Prediction and the forms of predictive imagining employed in contemporary environmental images (such as agriculture and climate research). While questions about the "geopolitics of planetary modification" 6 have become a central aspect of how we think of the ontologies of materiality and the Earth as Kathryn Yusoff has demonstrated, we are interested in how these materialities are also produced on the level of images.

Real time data processing of the Earth not as a single view entity, but an intensively mapped set of relations that unfold in real time data visualisations becomes a central way of continuing the earlier more symbolic forms of imagery such as the Blue Marble. ${ }^{7}$ Perhaps not deep time in the strictest geological terms, agricultural and other related environmental and geographical imaging are however one central way of understanding the visual culture of computational images that do not only record and represent, but predict and project as their modus operandi.

\footnotetext{
${ }^{5}$ See Joanna Zylinska, Nonhuman Photography, Cambridge (MA) 2017.

${ }^{6}$ Kathryn Yusoff, The Geoengine: Geoengineering and the Geopolitics of Planetary Modification, in: Environment and Planning A 45, 2013, S. 2799-2808.

${ }^{7}$ See also Benjamin Bratton, What We Do Is Secrete: On Virilio, Planetarity and Data Visualisation, in: John Armitage/Ryan Bishop (Hg.), Virilio and Visual Culture, Edinburgh 2013, S. 180-206, hier S. 200-203.
} 
This text will focus on this temporality of the image that is part of these techniques from the microtemporal operation of Next Frame Prediction to how it resonates with contemporary space imaging practices. While the article is itself part of a larger project where we elaborate with theoretical humanities and artistic research methods the visual culture of environmental imaging, we are unable in this restricted space to engage with the multiple contexts of this aspect of visual culture. Hence we will focus on the question of computational microtime, the visualized and predicted Earth times, and the hinge at the centre of this: the predicted time that comes out as an image. The various chrono-techniques ${ }^{8}$ that have entered the vocabulary of media studies are particularly apt in offering a cartography of what analytical procedures are at the back of producing time. Hence the issue is not only about what temporal processes are embedded in media technological operations, but what sounds like merely a tautological statement: what times are responsible for a production of time. What times of calculation produce imagined futures, statistically viable cases, predicted worlds? In other words, what microtemporal times are in our case at the back of a sense of a futurity that is conditioned in calculational, software based and dataset determined system?

\section{Planetary Images}

As an aftereffect of $20^{\text {th }}$ century rocketry, from V-2 to later abundance of satellites, the Earth is constantly visible. Besides often described as forms of optical visuality for example the terminology of overexposure as with Virilio ${ }^{9}-$ it is also a matter of calculation of visibility, and hence a central theme in past years of media theoretical discussions. As Jennifer Gabrys points out: "The architectures and algorithmic processes for relating sense data are a critical part of how sensor systems operate. They articulate how sense data will come together into arrangements indicative of environmental and planetary processes."10

Planetary visibility is calculated and composed in mathematical ways that have for a longer time already introduced a form of imaging that escapes the usual assumption about representationality. Steve Graham argues that the satellite produced orbital image must be understood both through its infrastructures (some of which is on the ground level) and how they constantly compose and produce the Earth's surface through and as an image.

Instead of invoking satellites as an absolute form of imperial vision, it is necessary, rather, to see satellite imagining as a highly biased form of visualizing or even simulating the earth's surface rather than some objective or

8 Wolfgang Ernst, Chronopoetics. The Temporal Being and Operativity of Technological Media, London-New York (NY) 2016.

${ }^{9}$ Paul Virilio, The Overexposed City, in: The Lost Dimension, New York (NY) 1991, S. 9-28.

${ }^{10}$ Jennifer Gabrys, Program Earth: Environmental Sensing Technology and the Making of a Computational Planet, Minneapolis (MN) 2016, S. 42 
apolitical transmission of its 'truth'. 11

The calculational basis of simulated earth's surface is subject to microtemporal operations that define it less as visual images than as statistical distributions even if they come out as temporal image series.

Indeed, as Peter Krapp argues relating to the planetary networks of knowledge concerning polar regions, the image of the planet as a holistic entity of knowledge is underpinned by the issue of scale - both spatial and temporal - that includes in this sense the particular microtemporality of the calculations that constantly compose and recompose the planet in its projected sense. ${ }^{12}$

Satellite images become one backbone of the temporality of the planetary view. From an assumption of an eye in the sky, the more accurate way to understand the potential of images is to think of them as part of "diachronic omniscience", to use Lisa Parks' formulation:

Archives of satellite image data thus create the potential for diachronic omniscience - vision through time - because they enable views of the past (and future with computer modelling) to be generated in the present that have never been known to exist at all, much less seen. Our understanding of the temporality of the satellite image should be derived through the process of its selection, display, and circulation rather than formed at the instant of its acquisition. $^{13}$

Such images are revealed primarily as datasets; agricultural growth, deforestation, ice retreat and other surface level transformations become integrated through rather mundane techniques that already speak to this transformation of both images and satellite data. Still images shift to moving images that track changes:

For a long time Earth observation was about the identification of specific features on an individual "still" image, starting with defense and security purposes, and then slowly spreading to other industries as well. While the stills part is true even today, with the introduction of ongoing monitoring programmes such as Landsat and Sentinel a new methodology appeared-the long-term observation of changes, developments, or simply monitoring vegetation growth. Having weekly (or better) coverage available over land worldwide makes it a uniquely powerful dataset. ${ }^{14}$

${ }^{11}$ Steve Graham, Vertical. The City from Satellites to Bunkers, London-New York 2016, S. 31.

${ }^{12}$ Peter Krapp, The Invisible Axis. From Polar Media to Planetary Networks, in: Nicole Starosielski/Janet Walker (Hg.), Sustainable Media, New York (NY) 2016, S. 264-279.

${ }^{13}$ Lisa Parks, Cultures in Orbit, Durham 2005, S. 91. Parks has also recently recalled the notion of diachronic omniscience in her approach to predictive analytics, war and satellite imagery in Lisa Parks, Rethinking Media Coverage. Vertical Mediation and the War on Terror, New York (NY)-London 2018, S. 131-136.

${ }^{14}$ Grega Milcinski, Multi-Year Time Series of Multi-Spectral Data Viewed and Analyzed in Sentinel Hub, in: https://medium.com/sentinel-hub/multi-year-time- 
Historical datasets are instrumentalised as predictive patterns that can be updated in real time. The question of earth (surface) time condenses in the moment of calculation that is open to real-time input from various data sources considered relevant. Geological and geographical times are not merely presented, but forwarded towards various speculative futures.

One key technique that we are interested in this context is "video frame prediction", which attaches these images to the legacy of moving images while also signaling their relevancy in contemporary cultures of data. The revisualization of archived images about environmental changes as time-lapse type video sets the ground for video prediction techniques that, as it will be shown next, are both part of the regime of digital images (codecs and compression) and of this large scale planetary imagining.

\section{Video Prediction}

Next Frame Prediction, or broadly speaking video prediction, names a strand of machine learning aimed at the algorithmic continuation of a given video. Explored so far in computer science environments, its main objective is the generation of a few frames of a yet unseen future: to foresee the next movements of the vehicles on the road in a self-driving situation ${ }^{15}$, for instance, or to envision the implications of the actions of a robotic arm in a room full of objects. ${ }^{16}$ Defined as the set of techniques aimed at the procedural continuation of videos with future frames, video prediction is therefore an activity aimed mainly at the generation of images. That is, it is not about the prediction of events only, but about the generation of visual appearances. This imaging ability, addressed in the technical literature as a "visual imagination of different futures" $" 17$ or as a "visual hallucination of probable events", ${ }^{18}$ is here a central aspect of what we wish to emphasize. While other machine learning developments related to video are meant to extract data in terms of classification, identification of objects, places or gestures within video streams ${ }^{19}$, Next Frame Prediction is an

series-of-multi-spectral-data-viewed-and-analyzed-in-sentinel-hub-5628ec4fad9c

[Zugriff 29.08.2018].

${ }^{15}$ William Lotter/Gabriel Kreiman/David Cox, Deep Predictive Coding Networks for Video Prediction and Unsupervised Learning, in: International Conference on Learning Representations, 2017 in: https://openreview.net/forum?id=B1ewdt9xe [Zugriff 29.08.2018].

${ }^{16}$ Chelsea Finn/Ian Goodfellow/Sergey Levine, Unsupervised Learning for Physical Interaction through Video Prediction, in: Advances in Neural Information Processing Systems 29, 2016, S. 64-72.

${ }^{17}$ Ebenda, S. 64.

${ }^{18}$ Jacob Walker/Abhinav Gupta/Martial Hebert, Patch to the Future: Unsupervised Visual Prediction, in: Computer Vision and Pattern Recognition, March 2014, S. 3302-3309, hier S. 3302.

${ }^{19}$ See, as examples of these, Google Research's project on video classification, AbuEl-Haija/Nisarg Kothari/Joonseok Lee/Paul Natsev/George Toderici/ Balakrishnan Varadarajan/ Sudheendra Vijayanarasimhan, YouTube-8M: A Large-Scale Video Classification Benchmarking, in: https://arxiv.org/pdf/1609.08675.pdf [Zugriff 29.08.2018] or the Places dataset 
operation focused on the generation of new images, to be used as plausible futures frames. In other words, it produces images that could be, that might take place, that relate to the ways movement moves in real space but calculated in microtemporal computational space. Hence, there is something interesting going on when one starts to consider the status of what these images are. The epistemology of images relating to classification and identification (e.g. from satellite imagery) is complemented by this particular video 'art' of prediction - images that could be, might be, and in any case, are valuable as hallucinations of future.

In order to work, machine learning based video prediction relies on a computational object -the model- that, given a sequence of frames as input, is able to produce as an output a subsequent set of frames. As it occurs characteristically in the context of artificial intelligence, the predictive model is built recursively in a so-called training process, where an initially randomized instance of it is gradually tuned to fit its function. Fed with a dataset of similarly looking videos, the training process goes through the videos in the archive and performs prediction tests. Training tests are calculated on sequences where the real next frame is known. The predicted frames are compared to the so-called ground truth, an actual video image in this case, in a way that the differences between the two are used to automatically modify the evolving model. This procedure is repeated then an appropriate number of times, so that the differences between the predictions and the ground truth are minimized.

In order to understand better the nature of the imaging capability of video prediction models, we will next consider two significant preconditions it relies on. As we will see, it is necessary to understand some key components about what are digital moving images as a way to understand what these machine learned images are.

First, Next Frame Prediction is based on the calculability of video frames. In order to be able to track and register the differences between frames, they need to be addressed as digital objects. As it has been extensively discussed ${ }^{20}$, frame difference is not just one of the possible numerical operations between frames, but the basic one that grounds the video encoding software, codecs, that allows the circulation and streaming of digital video. In digital video, frames are encoded in relation to their differences with their immediate past and future neighbours. They are not images in the sense of a classical optical photographic picture but aggregates of different moments of time that the decoding player assembles later for the human eye. ${ }^{21}$ Digital video relies on a microtemporal architecture where even the future is structurally anticipated. While there would be a lot more to discuss about the frame - and this is where cinema scholars and art historians could excel - suffice it to note in this short article that one key aspect relates then to the expression of movement as actual and

project elaborated to allow machine learning to infer the locations were sequences were shot: Bolei Zhou/Agata Lapedriza/Aditya Khosla/Aude Oliva/Antonio Torralb, Places: A 10 million Image Database for Scene Recognition, in: IEEE Transactions on Pattern Analysis and Machine Intelligence 40/6, 2017, S. 1452-1464.

${ }^{20}$ Adrian Mackenzie, Codecs in: Matthew Fuller (Hg.) Software Studies. A Lexicon, Cambridge (MA)-London, 2008; Sean Cubitt, The Practice of Light. A Genealogy of Visual Technologies from Prints to Pixels, Cambridge (MA) 2014; Ernst, Chronopoetics, wie Anm. 8.

${ }^{21}$ Ernst, Chronopoetics, wie Anm. 8, S. 156. 
virtual. Frames contain worlds, actual and virtual. In Cubitt's words: "The actual always contains in itself the virtual: every motion contains in itself the possibility of unforeseen development, only one of which becomes actual in the next frame, but all of which lie latent in the first". 22

On top of that, motion becomes embedded in the calculated workings of the frame. To make the predictive disposition of video encoding techniques even more explicit, a standard and ubiquitous compression algorithm relies on "motion prediction", a block-based technique that replaces most of the visual data by motion vectors ${ }^{23}$. Taking this into account, machine based video prediction -namely, a convolutional ${ }^{24}$ technique built on the escalation of frame differences- follows up the predictive condition of codecs. As a matter of fact, one of the typical approximations to this type of video prediction is the so-called "encoder-decoder" ${ }^{25}$ pair, where encoding is used as a reference to the activity performed by the algorithm. A common ground of temporal architectures underlies encoding and video prediction, both future-making techniques that stem from the calculational image.

The second requirement of Next Frame Prediction is the dependence on datasets with sequences that unfold temporally in a patterned and replicable way. The videos provided by traffic cameras or by simple procedural animations are examples of these. A neural network learns the data by approaching it as a set of training examples and then adjusting its internal model ${ }^{26}$. In the case of video prediction, the model is said to "capture the motion" ${ }^{27}$ that the videos display by training its predictive capabilities across the sequences in the dataset. Aptly enough, "motion capture" has its own media archaeological reference point in the early experiments with moving images: note here for instance Edward Muybridge's late $19^{\text {th }}$ century techniques for stopmotion and extracting data from the images and their intervals.

Of course, considering the context of data images in contemporary culture, the extractive process occurs in this case as a generalisation from examples. This relates then to a different sort of a media historical lineage than one solely about images; in other words, we are also dealing with statistics. Machine learning, sometimes referred to as statistical learning ${ }^{28}$, has been located at the intersection of computer science and

\footnotetext{
${ }^{22}$ Sean Cubitt, Finite Media. Environmental Implications of Digital Technologies, Durham-London 2017, S. 3.

${ }^{23}$ Mackenzie, Codecs, wie Anm. 20, S. 52-54.

${ }^{24}$ Convolutional accounts in the context of machine learning to the mathematical sort of scanning method that allows to address the spatial arrangement of the information in the image.

${ }^{25}$ Lotter/Kreiman/Cox, Deep Predictive, wie Anm. 15, S. 5.

${ }^{26}$ Adrian Mackenzie, Machine Learners. Archaeology of a Data Practice, Cambridge (MA) 2017, S. 27.

${ }^{27}$ Xingjian Shi/Zhihan Gao/Leonard Lausen/Hao Wang/ Wai-kin Wong/Wang-chun Woo/Dit-Yan Yeung, Deep Learning for Precipitation Nowcasting: A Benchmark and A New Model, in: Advances in Neural Information Processing Systems 30, 2017, S. 5617-5627, hier S. 5619.

${ }^{28}$ Mackenzie, Machine Learners, wie Anm. 26, S. 30.
} 
statistics $^{29}$, which itself would be a further media archaeological theme to investigate considering the roots of this mode of production of truths. ${ }^{30}$ Statistical methods are essential to the workings of these algorithms, whose capability to recognise behavioural patterns relies on a form of inductive inference linked to the contexts of data mining, as Louise Amoore has pointed out ${ }^{31}$. Future frames in video prediction are then inferred in a similar way as products are recommended to buyers by predictive analytics; following local correlations between individual items rather than global averages of historical data. Small bits of motion -the displacement of a car in movement or the enlargement of a tree in the street-are seized as recognizable patterns. The environment in motion becomes one part of the environment of images as part of environments of predictive calculation. The calculated images, then, generated along a microtemporally structured axis of time, piece together the inferred blocks of motion out of the dataset. Any temporal sequence of frames is therefore split by the model into a chained aggregation of recognized behaviors, and advanced as such - even if some don't actually exist, practicing apophenia when needed ${ }^{32}$. Worlds are created out of statistical data and out of controlled hallucination.

\section{From Next Frame Prediction to Numerical Weather Prediction}

In order to further elaborate on the implications of the statistical context of the timecalculated image, we are going to address next one of the first known environmental applications of these techniques - weather nowcasting. So far, we have argued that image-based machine learning has extended what Virilio called "environment control" as one form of temporal manipulation related also to both photographic and moving images. Video prediction includes the technical - even aesthetic - form of approaching also environmental changes, whether short term or longer term. While space imaging industry is already offering machine learning services fed with satellite imagery to forecast crop figures, retail sales and oil reserves information ${ }^{33}$, the generation of future images as performed by video prediction is still an experimental practice when dealing with environmental monitoring and forecasting duties. A

${ }^{29}$ See Martin van Otterlo, The Libraryness of Calculative Devices, in: Louise Amoore/Volha Plotukh (Hg.), Algorithmic Life. Calculative Devices in the Age of Big Data, New York (NY) 2016, S. 35-54, and, in the technical literature, Stefan Strauß, From Big Data to Deep Learning. A Leap Towards Strong AI or >Intelligentia Obscura $<$, in: Big Data and Cognitive Computing 2/16, S. 4.

${ }^{30}$ Louise Amoore, The Politics of Possibility. Risk and Security Beyond Probability, Durham 2013, S. 44 f.

${ }^{31}$ Ebenda, S. 41.

${ }^{32}$ On apophenia and algorithmic calculation see Amoore and Piotukh, Algorithmic Life, wie Anm. 29, S. 6 and Hito Steyerl, A Sea of Data. Apophenia and Pattern (MisRecognition) in: e-flux 72, April 2016; https://www.e-flux.com/journal/72/60480/asea-of-data-apophenia-and-pattern-mis-recognition/ [Zugriff 29.08.2018].

${ }^{33}$ See Planet Labs, Orbital Insight, Descartes Lab or Earth Now as examples of companies offering imaging services together with machine learning predictive analytics. In this vein, on the implications of artificial intelligence and predictive analytics on mapping techniques see Shannon Mattern, Mapping's Intelligent Agents, in: Places Journal, September 2017; https://doi.org/10.22269/170926 [Zugriff 29.08.2018]. 
significantly advanced context of application of these, however, is meteorology, where ongoing research has shown how Next Frame Prediction outperforms other technologies. In particular, when facing the problem of forecasting the immediate evolution of clouds over a specific location, video prediction has produced the most accurate predictions so far $^{34}$. This pilot application of environmental monitoring will allow us next to expand on the argument that closed the precedent section: the fact that environments of images structure also the temporality of the predicted image.

The accidental appearance of recognizable cloud formations in aerial photographs obtained with V-2 rockets spurred the meteorological interest in orbital imagery. Until the emergence of computing in the context of Cold War period, the meteorological now-moment had been produced in data maps reliant on a technical infrastructure of managing information, from the individual technologies of barometers to the networks over vast geographical areas over the telegraph. ${ }^{35}$ In contrast to the data grids, the orbital imaging infrastructure allowed to picture from above and at once large-scale systems, such as storms and other patterned structures. Orbital photomosaics attained additionally greater detail when compared to "the detection threshold of the data network" "36, giving rise, as environment historian Sebastian V. Grevsmühl has argued ${ }^{37}$, to a new visual language to address cloud formations and dynamics. In Paul Edwards' words, meteorologists started to "literally see large-scale weather systems, instead of laboring to construct maps and mental images from instrument readings alone." ${ }^{38}$ Since then, weather has been embedded in the planetary-scaled imaging infrastructure of satellites, telecommunications and processing stations.

In terms of techniques of future, however, despite the prominence of images as sources of information, the developments in computer technology kept physics-based data and models as the central predictive tools. Recalling Peter Galison's distinction between image and logic epistemic traditions in science ${ }^{39}$, forecasting was dominated in this case by the logic one, thanks to the early successes of calculative modelling. As a matter of fact, as Edwards argues, Numerical Weather Prediction (NWP) was rendered objective $-\mathrm{a}$ 'mature science' - against the inferred-subjective predictions based on data images ${ }^{40}$ : "for the first time, a weather forecast could be calculated objectively (at least in principle) from basic physical laws, rather than inferred from maps on the basis of subjective experience." ${ }^{41}$ Images, Virilio's real-time interfaces, replaced the position of sensor grids as builders of the now-moment of the weather reports. They rendered weather as a controllable and calculable object - but didn't

\footnotetext{
${ }^{34}$ Shi et al., Deep Learning for Precipitation Nowcasting, wie Anm. 27.

${ }^{35}$ Paul N. Edwards, A Vast Machine. Computer Models, Climate Data, and the Politics of Global Warming, Cambridge (MA)-London 2013, S. 40 f.

${ }^{36}$ Sebastian V. Grevsmühl, Serendipitous Outcomes in Space History. From Space Photography to Environmental Surveillance, in: Simone Turchetti/Peder Roberts (Hg.), The Surveillance Imperative, New York (NY) 2014, S. 171-191, hier S. 179.

${ }^{37}$ Ebenda, S. 176.

${ }^{38}$ Edwards, A Vast Machine, wie Anm. 35, S. 219.

${ }^{39}$ Peter Galison, Image and Logic. Material Culture of Microphysics, Chicago 1997.

${ }^{40}$ Edwards, A Vast Machine, wie Anm. 35, S. 85-88.

${ }^{41}$ Ebenda, S. 86.
} 
actually calculate it. Until recent developments in computer vision and machine learning, therefore, forecasting belonged to the data realms of numerical modeling.

The operational modifications of the image due to machine learning have caused meteorological changes to be not only observable but also predictable as images. As it has been shown recently ${ }^{42}$, the current experimental state of video prediction techniques has already improved the forecasting capabilities achieved by Numerical Weather Prediction. The evolution, movements and dynamics of clouds, observed and collected as sequences of images, have been used to train Next Frame Prediction models that are able now to produce one to six hours of visual forecasts. Cloudy skies of the near future are thus predicted and envisioned, without any reference to physicsbased models or magnitudes. As expressed in the scientific literature, "precipitation nowcasting and video prediction are intrinsically spatiotemporal sequence forecasting problems in which both the input and output are spatiotemporal sequences"43.

Nowcasting, a term familiar both to meteorology as well as economics, comes out as a practice of infrastructural imaging. The very act of monitoring and systematically registering entails a calculable future. Sequences of images give rise to models without the need to refer to any previous scientific diagram. Orbital monitoring operations constituted environmental imaging as a twofold operation: on the one hand the environment was imaged, on the other, environments of images resulted. Archiving, processing and circulating, these environments of images reveal -when performed as machine learning- the variations and modulations we perceive as time. Inferred futures, technically hallucinated, are the legacy of the contemporary calculational infrastructures of space imaging.

\section{Conclusions}

"A hallucination is neither metaphor nor imagination but the reappearance in the real of a signifier that was rejected in the symbolic" ${ }^{44}$ writes Bernhard Siegert allowing us to pick up on the otherwise perhaps casual engineering reference to "visual hallucination of probable events" that we quoted above in passing. From the symbolic representations of the planetary entity to the real-time projections about the future, forms of future-making have been part of the geographical and geological visualization that is of significant economic and security related importance. The symbolically significant futures presented in genres such as science fiction or other forms of future-making are complemented by the constant - and grey - operations of prediction.

Our article has engaged with video frame prediction and nowcasting but of course these need to be set as part of a bigger picture of both calculation and the temporality of images that are central part of contemporary culture. An important question

\footnotetext{
${ }^{42}$ Shi et al., Deep Learning for Precipitation Nowcasting, wie Anm. 27.

${ }^{43}$ Ebenda, S. 3.

${ }^{44}$ Bernhard Siegert, Eating Animals - Eating God - Eating Man. Variations on the Last Supper, or, the Cultural Techniques of Communion, in: ders., Cultural Techniques. Grids, Filters, Doors, and Other Articulations of the Real, New York (NY) 2015, S. 33-52, hier S. 51.
} 
emerges: what are the forms of time that are part of this infrastructure of calculation and how do they still operate as images? While images are primarily addressable as files, formats, and data, their microtemporal operations produce other times too: times of projection, prediction and forecasting. It is this bundle of questions about images, time and machine learning that we wanted to present briefly in this article while acknowledging that the question is both technically much bigger in terms of the planetary infrastructures and conceptually much far-reaching as it deals with a variety of questions of temporality and techniques of geographical and geological surfaces. While we started with Virilio's at one point significant take on the repercussions of vision machines, this must be expanded to include the techniques of images produced out of statistical inference. Inferred and envisioned, futures form as part of the production of images. 